\title{
Evaluation of the preoperative preparation effectiveness with the ozone therapy and NPWT usage in patients with pressure sores after spinal cord injuries.
}

\author{
Igor Plis, Yaroslav Zarutskii
}

\section{CASE REPORT}

\begin{abstract}
Surgical treatment of 178 patients with pressure sores in the rehabilitation period of traumatic illness was analyzed and a surgical treatment with the use of the NPWT technique in the preoperative period was suggested. A positive effect of NPWT on local microcirculation, neoangiogenesis, bacterial decontamination and tissue repair was observed. The indices of immunohistochemical markers CD34, CD68, which reflect the degree of reparative processes in the wound, were determined. The increase in the density of the CD34-expressed vessels and the quantitative index of macrophages CD68+ ( $40.8 \pm 1.7 \%$ ), with the minimal advantage of the functionally active macrophages are the objective criteria of tissue readiness for plastic closure. The use of (local) musculocutaneous flaps with axial blood supply in combination with anatomical and functional resection of the underlying bones was suggested as a method of plastic surgery of pressure-trophic ulcers, which reduced the number of early postoperative complications. Good and satisfactory results were achieved in $109(90.8 \%)$ patients.
\end{abstract}

Keywords-pressure ulcer, NPWT, microcirculation, immunohistochemistry

\section{INTRODUCTION}

$\mathbf{T}$ HE frequency of progression or pressure sores in the injured patients with the spinal cord injury ranges from $15-75 \%$. 2 Disruption of innervation, tissue tropism and microcirculation complicates the implementation of plastic reconstructive surgery, the patients require long preoperative preparation and careful follow-up in the postoperative period. ${ }^{[3}$ Surgical treatment of pressure sores is a promising area, but the high level of postoperative complications (divergence of seams $10-25 \%$, suppuration $50-75 \%$, fistula formation $38-85 \%$ ) causes a low surgical activity 3 , 6 , 7

In recent years, considerable attention has been paid to the investigation and recovery of the microcirculatory bed in the treatment of pressure sores, which is the decisive factor in the healing of such wounds 589 Informative indices of the intensity of reparative processes in soft tissues are microcirculation, tissue saturation, endothelial markers of vessels

Manuscript received 27.02.2018; revised 12.07.2018. This work did not receive any financial support. Author declares no conflict of interest.

Author affiliations: Military Hospital, Mukachevo , (IP) ; Ukrainian Military Medical Academy, Kyiv, (YZ)

*Correspondence to: Igor Plis: docplis30@yahoo.com
(CD34), macrophage markers (CD68). In recent years, data on the leading role of macrophages in tissue repair due to the production of fibroblast growth factors (FGF), granulocytemacrophage-colony-stimulating factor (GM-CSF), plateletderived growth factor (PDGF), transforming growth factor (TGF) became available, which provide the organization of extracellular components matrix and the transition of the wound process to the regeneration phase $5.80,10,11$

The scientific literature describes the effective use of ozonotherapy in patients with purulent complications of wounds. The therapeutic application of ozone can have a profound antibacterial effect, perform anti-inflammatory and immunomodulating action, improve blood rheology, promote the normalization of microhemodynamic disorders, and help to correct the violations of the normal ratio of lipid peroxidation processes and the activity of the antioxidant system, which is of great clinical importance in the treatment of surgical infections.

A promising area in the treatment of patients with pressure sores is the use of the negative pressure wound therapy - NPWT. The main effects of vacuum therapy are the active removal of wound exudate, maintenance of a moist environment, increased microbial decontamination of tissues, reduction of interstitial edema, contraction of the edges of the wound surface? 912 . 15

The purpose of the study was to investigate the peculiarities of the influence of ozone therapy, NPWT on the key indicators of tissue repair of pressure sores and to determine the objective indicators of soft tissue readiness to plastic surgery.

\section{A. Materials and methods}

The work is based on the results of examination and treatment of 178 patients with the consequences of vertebralspinal cord injury, which was complicated by the development of pressure sores in the rehabilitation period of traumatic disease. The criteria for inclusion of the injured to the study were:

- rehabilitation period of traumatic illness due to spinal cord injury; 


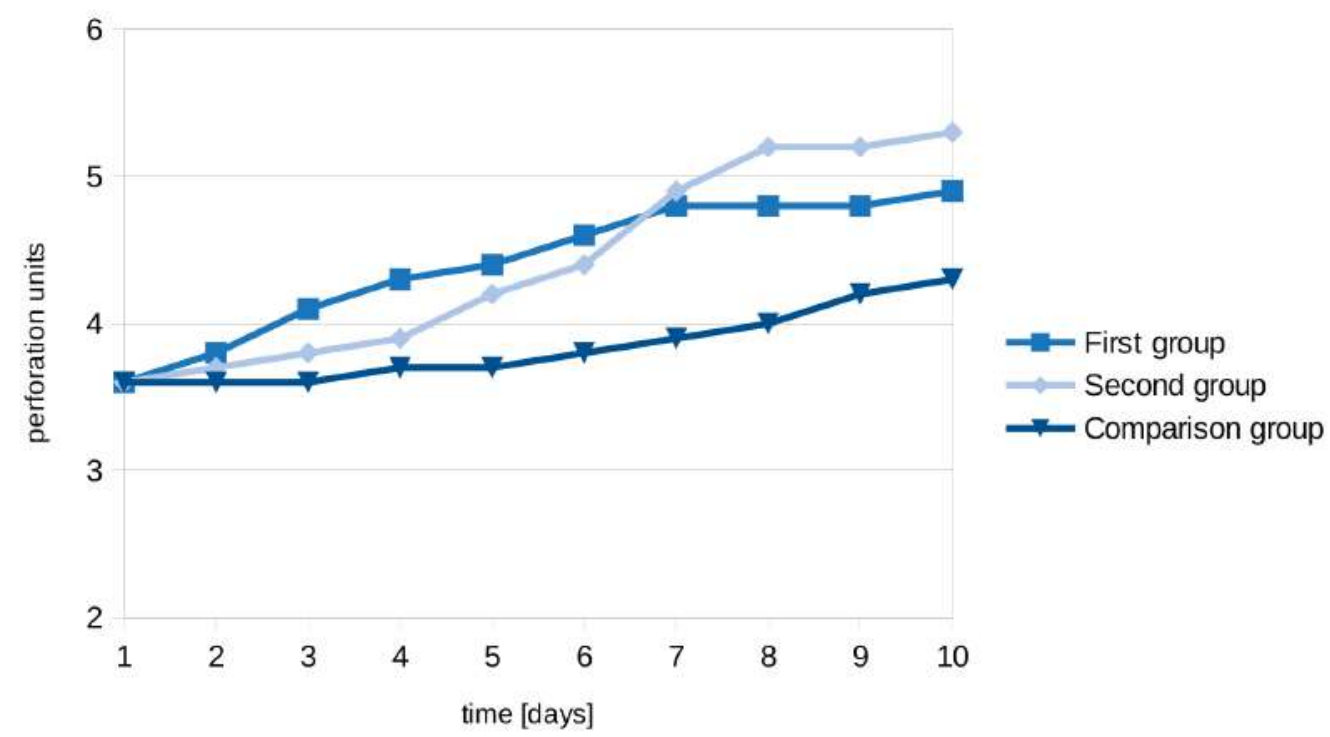

Figure 1. Dynamics of microcirculation rate in study groups.

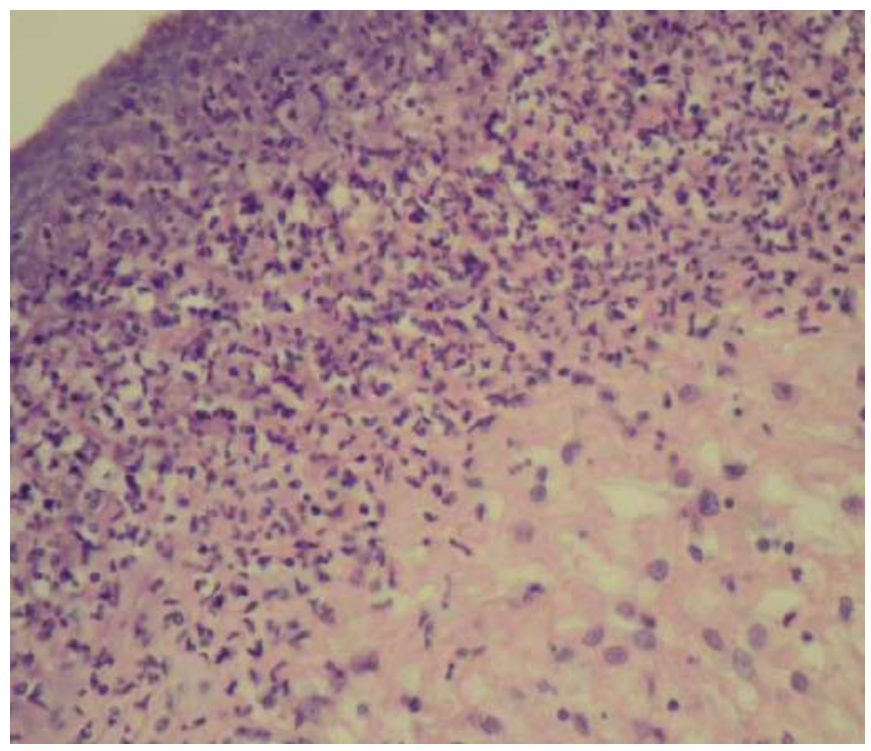

Figure 2. Fragment of pressure sores (epithelial dysplasia, nuclear detritus, fibrin). Staining with hematoxylin-eosin $\times 400$.

- localization of pressure sores in the sacral area, in the greater trochanter, in the area of ischial tuberosity;

- the existence of pressure sores for more than three months.

- patients with the diagnosed stage III-IV of pressure sores according to the Agency for Healthcare Policy and Research (1992) classification. The criteria for exclusion of the injured were the existence of:

- pressure sores of the other localization (lower leg, heel, foot);

Criteria for exclusion of the injured were:

- multiple pressure sores wounds;
- the unwillingness of the injured to participate in the research.

The research has been fulfilled on the bases of militarymedical institutions of Ministry of Defence of Ukraine during 2006 till 2017, and was subdivided into three phases, depending on the material and technical possibilities and abilities to implement modern technologies for the injured preoperative preparation to plasty.

During the first stage, from 2006 till 2008, the comparison group was formed and consisted of 58 injured, who underwent conventional preoperative preparation with the use of stage surgical treatments, daily dressings with solutions of antiseptics, ointment compositions, wound coverings. The first group has been formed since 2008, with the introduction of ozone therapy methods in medical institutions of Ministry of Defence of Ukraine. The first group included 76 patients who, together with stage surgical treatment of pressure sores, had general and local ozone therapy. The total ozonotherapy was carried out in the form of an injection of $200 \mathrm{ml}$ of ozonized physiological $0.9 \%$ sodium chloride solution at a rate of $80 \mathrm{ml} / \mathrm{min}$, concentration of 3-6 mg/l within 8-10 procedures. The "Ozone UM-80" medical device was used as an ozone generator. The cavities of the pressure ulcers were topically filled with sterile napkins moistened with an ozonized $0.9 \%$ physiological solution with a concentration of $15-20 \mathrm{mg} / \mathrm{l}$ and an exposure of 20 minutes. In parallel, the wound surface was gassed with an ozone-oxygen mixture in a plastic chamber with an ozone concentration of $10-15 \mathrm{mg} / \mathrm{l}$, a flow rate of $0.51 / \mathrm{min}$, for 20 minutes. In the case of weak dynamics of wound surface cleaning and the appearance of perifocal inflammation, signs of microbial colonization, the concentration of ozone in the mixture was increased to 20-30 $\mathrm{mg} / \mathrm{l}$ to enhance the bactericidal and necrolytic effect.

The second group included 44 injured, who were treated 


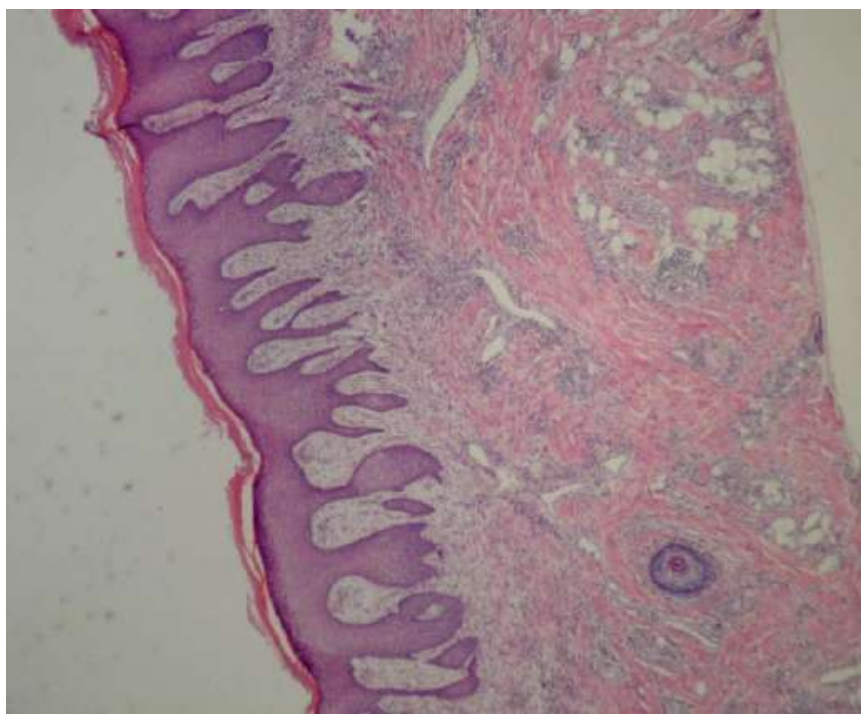

Figure 3. Fragment of the skin of the edge of pressure sores (acanthosis, lympho-histiocytic infiltration). Staining with hematoxylin-eosin $\times 400$.

with the NPWT method in low (down to $-75 \mathrm{mmHg}$ ) and medium (from -75 to $-120 \mathrm{mmHg}$ ) decompression range to prepare for plastic surgery. After the debridement, a polyurethane sponge with a perforated drainage inside modeled in shape and size was placed in the wound. In cases of deep wounds, they were tamponated with separate sponges with close contact with the sponge in the wound cavity and fixed to the edges with separate nodal sutures. In complex forms of defects, internal bridges were used, which densely contacted the main sponge. In cases of the presence of the underlying bone with signs of osteomyelitis in a bed of pressure sores, a decortication was made, followed by closure with a polyurethane sponge. This technique helped to eliminate foci of osteomyelitis, stimulated regional blood flow and growth of granulation tissue. The wound was covered from above with adhesive film to achieve complete tightness. The outer end of the drain was connected to a vacuum system at a constant aspiration mode. The change of dressings was carried out after 24-72 hours, depending on the nature of the course of the wound process, the amount and nature of the exudate isolated from the wounds.

Evaluation of the effectiveness of preoperative treatment was carried out based on the nature of the course of the wound-healing process, microbiological monitoring, determination of local microcirculation, morphological, immunohistochemical studies.

\section{B. Examination of microcirculation}

Local microcirculation parameters were determined by the LAKK-02 laser analyzer: the microcirculation index and the total saturation index have been measured by laser beam with a wavelength of 0.63 microns, to measure the microcirculation of blood. The skin was tested at a distance of $1 \mathrm{~cm}$ from the edge in patients with a state of complete physical and mental rest in a lying position, in a room with a temperature of $+22-24$ o, which has been maintained with the help of air conditioning system for 3 minutes. The state of the skin microcirculation of the lesion has been evaluated by the Laser Doppler Flowmetry (LDF) method twice: at the admission to the medical institution and at the 10th day of preoperative preparation. The LDF 2.20.0.507WL software for Windows was used to record and register the LDF-grams on the computer. LDF-gram is a resultant parameter determining the dynamic characteristics of blood microcirculation - changes in blood flow (tissue perfusion) per unit of time in a definite volume.

\section{Morphological study}

The tissues were the material for morphological studies, including the walls and bottom of the ulcers, granulation and scar tissues, the skin, adjacent to the edges of the ulcers, which were excised, during the first days after hospitalization, as well as after the training at 10th-14th day, as a single unit, intraoperatively. For observational light microscopy of pathological processes, the pieces of tissue have been used, measuring $1 \times 1 \times 0.5 \mathrm{~cm}$, which were fixed for 14 days in $10 \%$ neutral formalin solution $(\mathrm{pH}=7.0)$. The tissues were dehydrated in graded alcohols with subsequent paraffin blocks manufacture according to the generally accepted technique. Serial sections, in thickness of 5-8 microns, followed by haematoxylin and eosin staining, have been made from paraffin blocks.

\section{Immunohistochemical study}

Immunohistochemical study of tissue samples was carried out in a standardized manner using serial paraffin sections 3-5 $\mu \mathrm{m}$ thick, placed on adhesive glass and coated with polysine (Menzel-Glaser) and DAKO reagents with monoclonal antibodies CD68 (ClonePG-M1, dilution 1:50) and CD34 (CloneQbend10), Envision TM FLEX + visualization system, CodeK8012 on the DAKO auto-stainer. Evaluation of the level of cytoplasmic expression of CD34 was performed taking into account the intensity of color and distribution in endotheliocytes in 5 randomly selected fields of view of the microscope with a magnification of $\times 200$. The intensity of cytoplasmic expression of CD68 was evaluated by a semi-quantitative method on the basis of the color intensity and the number of granules in the cytoplasm, followed by their differentiation into macrophages with weak and pronounced expression. Viewing and digital photographs of micro-preparations were carried out with a digital camera "Olympus C 5050Z", on a microscope "Olympus CX-41".

\section{E. Evaluation of postoperative complications}

The early and late postoperative complications after plastic surgery, in order to evaluate the effectiveness of treatment in the studied groups of patients with pressure sores, have been traced and analysed. The results, up to 6 months, including the time of surgical operations, have been considered as the nearest ones, and have been classified as good, satisfactory and unsatisfactory. Good results were characterized by the complete replacement of the defect in the pressure sores 


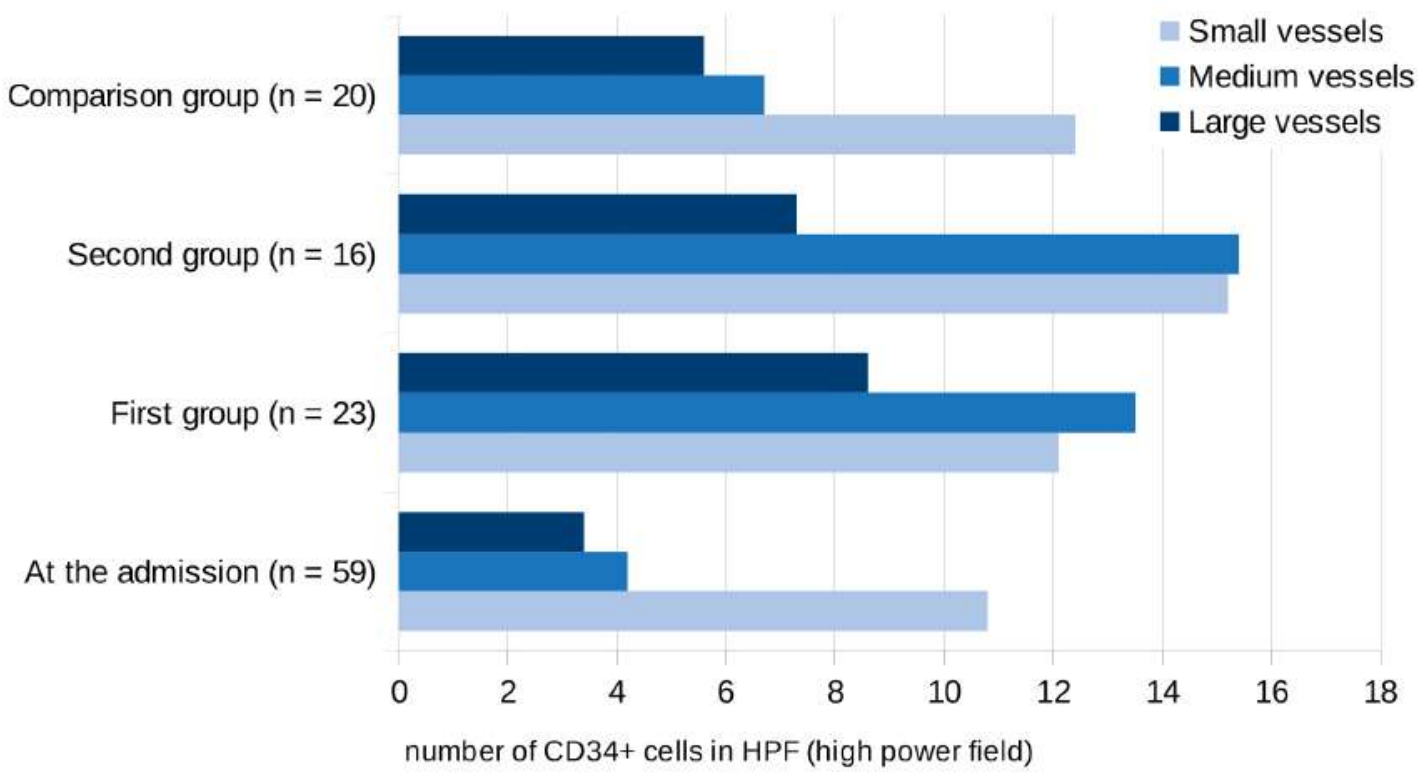

Figure 4. Distribution and density of expressed CD34 endothelial cells in pressure sore tissues

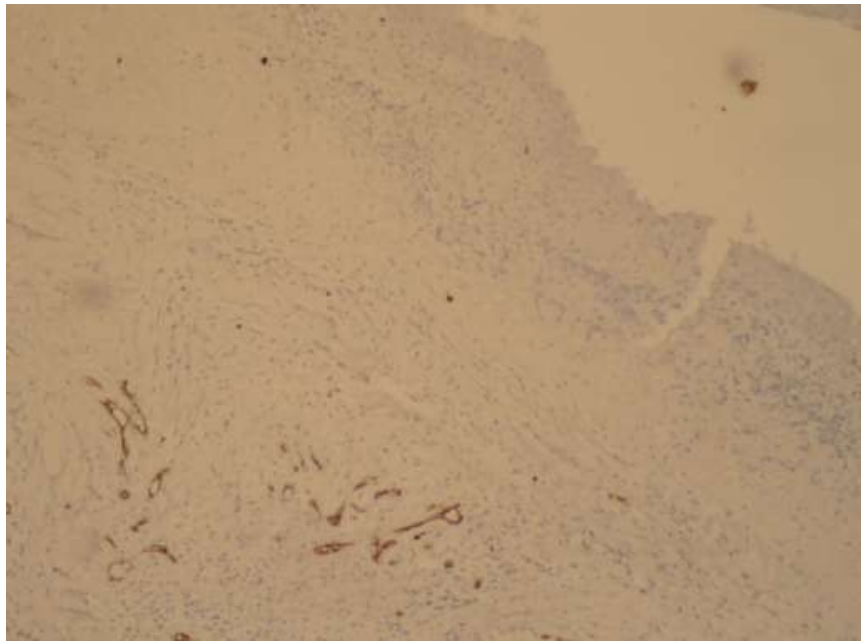

Figure 5. Expression of CD34 on endothelial cells in granulation tissue at the beginning of preparation. Simulated gas chromatography distillation. FLEX visualization system. Magnification: $\times 200$

area, the primary healing of the postoperative wound, as well as the absence of early postoperative complications. During the healing of postoperative wounds by secondary tension, cases of incomplete replacement of the defect, early postoperative complications, with complete wound closure were considered as satisfactory. The results of surgical operations that ended with necrosis of the flap, tissue defect preservation or enlargement were considered unsatisfactory. Long-term results have been evaluated after 6 months of follow-up of the postoperative wound in the affected. The absence of complications in the operation area and a complete restoration of the support ability of the operated area, were considered as satisfactory results. The relapse of pressure

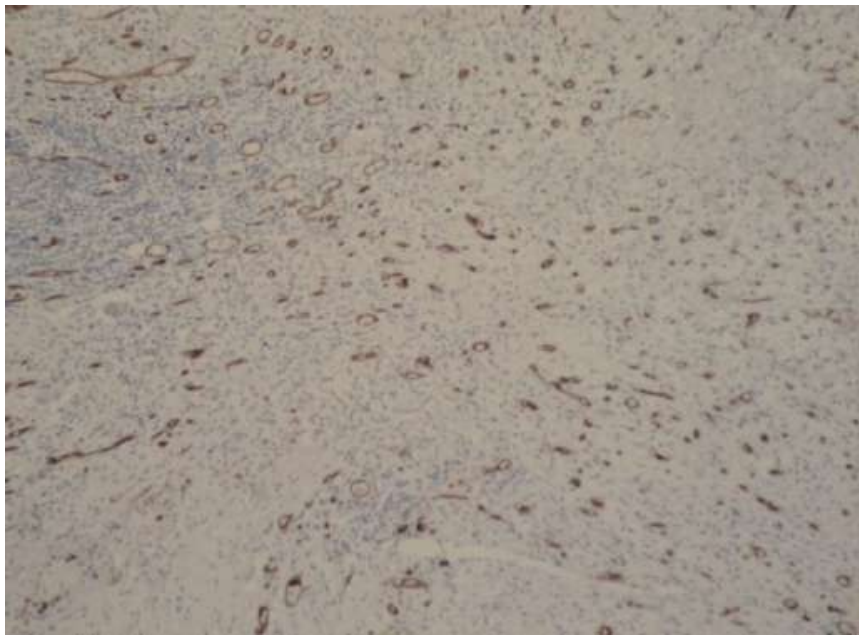

Figure 6. Expression of CD34 on endothelial cells in the deep layers of granulation tissue on day 10 , the second group. Simulated gas chromatography distillation. FLEX visualization system. Magnification: $\times 200$

sores, the appearance of fistulas in the area of postoperative wound, were considered as unsatisfactory results. For all the results of the study, statistical processing was carried out for parametric and nonparametric criteria using the "Medstat" program. Statistical differences between the groups were assessed according to Student's t-test by the definition of Chi-square. The reliability of the differences was determined for the significance level $\mathrm{P}<0.05$ (95\% significance level). The relationship between the investigated indicators was estimated from the results of the correlation analysis with the calculation of the Pearson and Spearman correlation coefficient. 


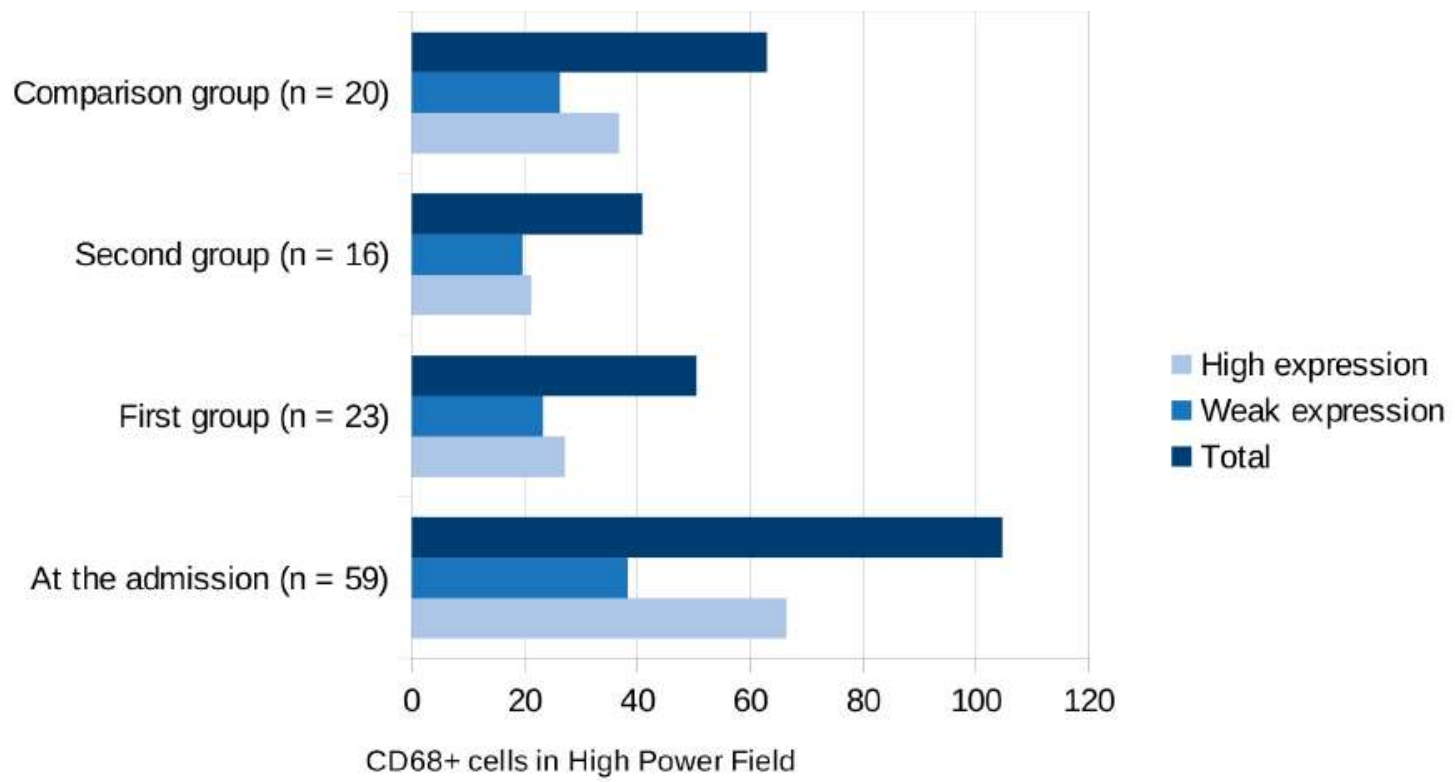

Figure 7. Distribution and intensity of expression of CD68 macrophages in biopsy specimens.

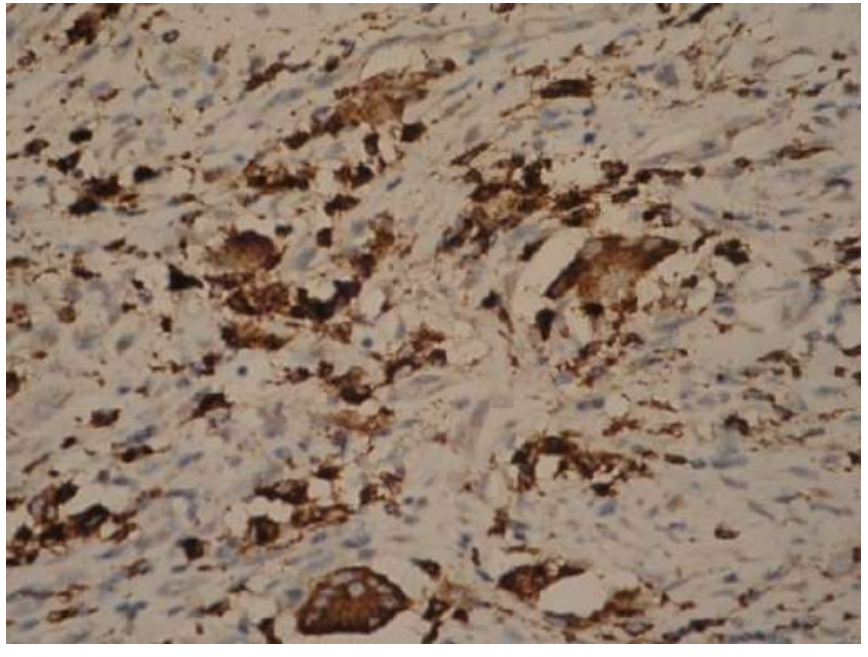

Figure 8. Fragment of the tissue of the pressure sore of the patient from the comparison group. Multinucleated giant cells (arrow). Simulated gas chromatography distillation. FLEX visualization system. Magnification: $\times 400$

\section{F. Results and discussion}

The majority of patients - $80.9 \%$ were from 20 to 50 years-old. The average age was $34.9 \pm 8.7$ years, men predominated $-147(82.6 \%)$. It has been revealed from the anamnesis that in $148(83,2) \%$ of injured pressure sores were formed as a result of a long seating in a wheelchair, that is, the pathological process began with tissue damage in the area of the underlying bones of the shank base and the subsequent transition of the necrotic process to the soft tissues and toward the skin covering. And even after the trophic disorders detection in this area, conducted conservative treatment and surgical interventions, the wheelchairs were actively used

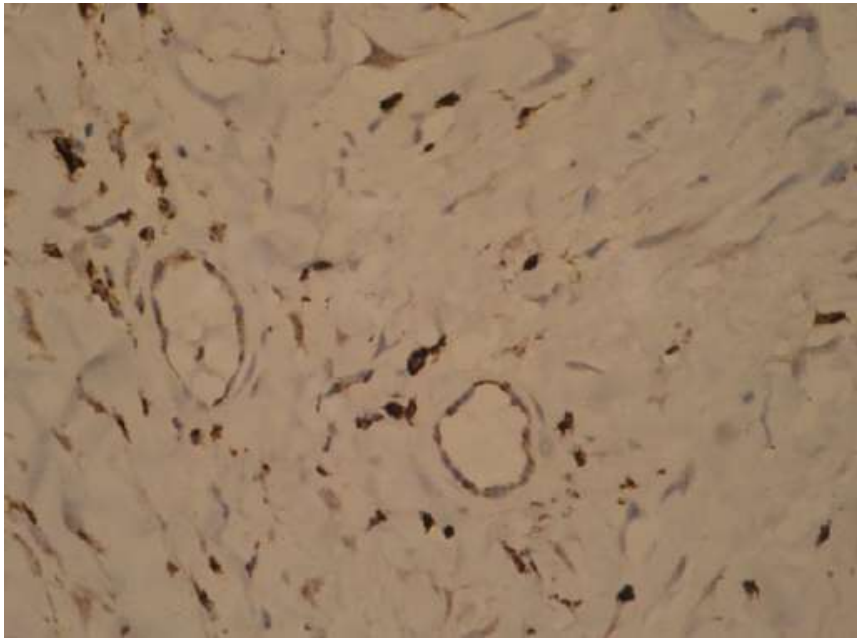

Figure 9. Expression of CD68 macrophages in surface granulation tissue The second group. Simulated gas chromatography distillation. FLEX visualization system. Magnification: $\times 400$

by injured, that is, the compression factor remained active. This led to continuously recurrent wound healing with the formation of chronic purulent complication of wounds, with osteomyelitis complicated underlying bone.

According to the Frankel / Asia classification, 135 (75.8\%) of the injured were assigned to group A, with no motor and sensory innervation below the site of spinal cord injury. Pressure sores were localized in the sacral area in 71 patients $(39.8 \%)$, in the area of ischial tuberosity in 58 patients $(32.6 \%)$, in the greater trochanter in 49 patients $(27.5 \%)$. Patients with pressure sores in other places and multiple pressure sores were not included in the study.

According to the Agency for Healthcare Policy and Re- 
search classification, 141 (79.2\%) patients were diagnosed with stage IV pressure sores, with complete loss of skin thickness, muscle necrosis, bone destruction. The stage III of pressure sores have been observed in 37 (20.8\%) patients and have been characterised by the destruction of the entire thickness of the dermis. Patients affected by pressure sores of I-II stages were not included in the study because such defects were not considered as indicators for plastic closure. During the analysis of the wounds, it has been found that pressure sores occurred mostly alongside with significant defects of soft tissues, the bottom of which were important anatomical formations (tendons, muscles, bones). Taking into account the fact that the injured were in their traumatic illness rehabilitation period, pressure sores has been observed as chronic fistulas with significant defects of soft tissues in their depths. The enlargement of the rough scar tissue around the ulcers significantly restricted the possibility of the wound self-healing and has been considered as an indicator for plastic closure. The duration of pressure sores ranged from 3 months to 42 years. In the comparative analysis of the pressure sores duration, it has been found that in $54(30.3 \%)$ patients, pressure sores lasted for more than three years and had the same frequency in all groups. Only in 34 (19.1\%) patients, we observed pressure sores that appear up to 6 months after the injury.

\section{G. Microbiological studies}

Analysis of the results of microbiological studies showed that the main microbiological pathogens in all study groups were staphylococci (37.2\%), E. coli (18.8\%), Pseudomonas aeruginosa (11.1\%), Proteus (10.3\%). Less common was Streptococcus pyogenes (8.4\%), Candida fungi (4.9\%). Microorganisms were isolated both in monoculture and in associations of staphylococcal flora with gram-negative flora (34.3\%), and Candida albicans (11.9\%).

The rate of decontamination was considered a predictive criterion for the successful preparation for the implementation of cutaneous plasty. On the 1st day of preoperative preparation, the number of CFU/g in the first, second and in the comparison group was equivalent. It was found out that on the 5th day there were significant differences in the contamination of wounds in the study groups.

The number of CFU/g in the first group decreased to 105 in $18(23.7 \%)$ and to 104 in $5(6.6 \%)$ of the injured. In the second group, the number of CFU/g decreased almost to 105104 in 18 patients $(42.2 \%)$. In the comparison group, only $14(24.1 \%)$ of injured had CFU/g decreased to 105-104 on the 5th day. On the 10th day in the first and second group, the level of contamination decreased by around 3-4 times in $65.7-81.8 \%$ of the affected patients, respectively, which allowed performing the surgical reconstructive operations while in the comparison group the critical level of contamination prevailed and only on the 12th day in 30 patients $(51.7 \%)$ the number of microbial cells in the wound dropped below the critical level. This confirmed the literature data on the bactericidal effect of ozone therapy and a significant reduction in bacterial contamination of wounds against the background of using NPWT.

\section{H. Examination of microcirculation}

The examination of microcirculation of the skin around pressure ulcers showed that $139(78.1 \%)$ patients in the course of hospitalization had a pronounced microcirculation disorders of the surrounding tissues, which was manifested by a decrease in microcirculation rate to $30.2 \%$ (up to $3.7 \pm$ 0,6 perf. units) in comparison with its normal values $(5.3 \pm$ 1.7 perf. units).

On the 10th day of preoperative preparation in the first group of patients, the microcirculation rate increased in all areas, maximally in the sacral area to $4.9 \pm 0.56$ perf. units. In the second group of patients, a significant increase in microcirculation rate was noted, even exceeding the normal value, maximally in the area of the ischial tuberosity to 5.3 \pm 0.62 perf. units, indicating the fullness of the microcytic channel due to the arterial component. In the comparison group, the microcirculation rate increased to $4.3 \pm 0.75$ perf. units.

The overall oxyhemoglobin saturation index did not differ significantly in clinical groups during hospitalization, and was determined at a level of $33.2 \% \pm 8.5$ to $37.4 \% \pm 7.5$. The growth rate of oxyhemoglobin saturation on the 10th day of preparation was more rapid in the first group with the maximum increase in the area of the ischial tuberosity to $58.1 \% \pm 8.5$, which was associated with the application of ozone therapy techniques. In the second group of patients on the 10th day, the saturation index in the sacral area was $51.6 \% \pm 11.3$, in the area of the ischial tuberosity $-55.8 \%$ \pm 10.3 , in the greater trochanter area $-53.1 \% \pm 11.5$. In the comparison group on the 10th day, the saturation index in the sacral area reached the values of $46.4 \% \pm 12.4$, in the area of the ischial tuberosity $-49.3 \% \pm 11.7$, and in the greater trochanter area $-45.7 \% \pm 12.4$.

These indicators of local microcirculation generally correlated with the clinical manifestations of the course of the wound process. Therefore, when choosing the method of pressure sore plasty, it is important to take into account the microcirculation data, especially when using poorly vascularized flaps in the case of autodermoplasty.

\section{Morphological study of the biopsy material}

In the morphological study of the biopsy material during hospitalization, pathological changes were observed, characteristic for long-term non-healing neurotrophic ulcers with the presence of a complex of alternative, exudative and proliferative reactions simultaneously. The epidermis was absent in ulcers, the wound surface was represented by a layer of leukocyte necrotic detritus, which consisted of a large number of segmented leukocytes, which were located among the fine-grained eosinophilic masses of necrotic cells, vessels, hyperchromic small clumps of nuclear detritus and fibrin (Fig. 2).

In biopsy materials of the skin on the border with a ulcerative defect the main changes were observed in the epidermis. It's thickening with hyperkeratosis and parakeratosis, pronounced acanthotic branched outgrowths in the dermis, proliferative activity of basal epitheliocytes was revealed. In 
Table I

DISTRIBUTION OF PATIENTS ACCORDING TO THE PERIODS OF PREOPERATIVE PREPARATION FOR DERMAL PLASTY (IN DAYS)

\begin{tabular}{llll}
\hline Method of preoperative preparation ((in days) & Sacrum & Ischial tuberosity & $\begin{array}{l}\text { Greater } \\
\text { trochanter }\end{array}$ \\
\hline First group (Ozone therapy) & $10.8 \pm 1.1$ & $13.1 \pm 1.2$ & $12.5 \pm 1.1$ \\
Second group (NPWT) & $9.3 \pm 0.9$ & $11.7 \pm 1.1$ & $10.2 \pm 0.9$ \\
Comparison group (Dressings and wound coverings) & $13.4 \pm 1.2$ & $18.5 \pm 1.5$ & $15.7 \pm 1.3$ \\
Total & $11.2 \pm 0.9$ & $14.4 \pm 1.2$ & $12.8 \pm 1.2$ \\
\hline
\end{tabular}

the papillary and reticular layer of the dermis, large foci of fibrosis and dense perivascular infiltrates were identified, represented by lymphocytes, macrophages, neutrophils and plasmocytes. Attention was drawn to the absence and reduction of the appendages of the skin, including the hair follicles (Fig. 3).

\section{J. Immunohistochemical study}

When the biopsy specimens were examined by immunohistochemistry on admission and taken intraoperatively on the 9th-12th day after the preparation, a positive immunohistochemical reaction with antibodies to CD34 was observed in the form of cytoplasmic expression of endothelial cells and is shown in (Fig. 4). As seen in (Fig. 4), in patients at the admission to the hospital, the positive reaction with the marker of angiogenesis CD34 was determined in $18.4 \pm 1.1$ vessels in the field of vision in the form of fine structures on intimal endothelial cells, which indicated poor activity of microangiogenesis processes. Small vessels up to $6 \mu \mathrm{m}$ predominated (Fig. 5).

The analysis of intraoperative biopsy specimens in the first group revealed an increase in the total number of vessels on the background of the preoperative preparation to 34.2 \pm 1.4 per HPF, with the predominance of medium forms (13.5 \pm 0.9 per HPF), located in groups, and large forms $(8.6 \pm 0.5$ per HPF). In the second group, the total number of vessels significantly increased to $37.9 \pm 1.8$ per HPF with a uniform advantage of small (15.2 \pm 0.9 per HPF) and medium (15.4 \pm 0.9 ) forms (Fig. 7). In the comparison group, the total number of vessels in intraoperative biopsies was $24.7 \pm 1.5$ per HPF, with the predominance of small $(12.4 \pm 0.8$ per HPF) granulation tissue capillaries in the foci of a chronic inflammatory infiltrate, surrounded by fibrous interlayers. Such indicators of angiogenesis, manifested by incomplete maturation and differentiation of blood vessels, are regarded as a result of insufficient effectiveness of preoperative preparation in the comparison group.

Immunohistochemical study of the distribution of CD68+ cells revealed two generations of macrophages: polymorphous small elongated macrophages with poor visualization of granules that had low functional activity, as well as highfunction large rounded macrophages with intense cytoplasmic granularity. The results of the distribution of CD68+ cells, depending on their functional activity, are shown in (Fig. 6).

The highest total pool of CD 68+ macrophages was observed at the beginning of preoperative preparation, the total number was $104.7 \pm 3.9$ per HPF, which was significant
( $p<0.05)$ with respect to the norm values $(10.6 \pm 1,1$ per HPF) and exceeded them by 10 times. Macrophages with a pronounced activity ( $66.4 \pm 2.5$ per HPF) with a functional activity index of 1.73 prevailed, which indicated the stress of the macrophage system in the focus of inflammation aimed at the active phagocytosis of necrotic detritus. In the first group of patients on the 10th-12th day the amount of expressed CD $68+$ cells was $50.5 \pm 2.3$ per HPF, of which $23.3 \pm$ 1.1 per HPF cells with weak functional activity and 27,2 \pm 1.3 per HPF cells with high functional activity, and the foci of weak staining were also determined in the walls of the vessels (Fig. 8). In the biopsies of the second group patients, the ratio of high- and low-functional macrophages was $21.2 / 19.6 \pm 0.9$ per HPF with a functional activity index of 1.08 , which was maximally approximated to the normal values of activity of reparative processes. In the comparison group using conventional preparation, the density of CD68+ expression decreased insignificantly, the total pool of macrophages was $62.9 \pm 1.9$ per HPF. The ratio of lowand high-functional cells was practically equal and amounted to $26.2 \pm 1.2$ and $36.7 \pm 1.4$, respectively. Another feature of the distribution of macrophages was the accumulation of large oval cells with a pronounced positive response, which were grouped and formed giant multinucleate cells that also expressed CD68.

The above morphological changes played a key role in triggering local immune responses that sustained chronic inflammation, impeded adequate regeneration processes in the source of destruction. The study revealed that at the admission the injured experienced hyper reaction of macrophages, which was accompanied by the formation of long-lasting non-healing ulcerative defects with a weak regenerative potential, on the other hand, anergy and depletion of the macrophage system, which led to disruption of the mechanisms of inflammation limitation and the progression of the purulent-destructive process. In the first and second group, the number of macrophages did not reach the normal values characteristic of intact skin, hence the reparative processes were not completed. However, the established quantitative indicator of CD68+ macrophages was $40.8 \pm 1.7$, with a minimal advantage of functionally active macrophages (functional activity index 1.08), which can be considered a significant diagnostic criterion in assessing the effectiveness of preparation, indicating an adequate healing and restoration process of structural and functional components of the skin, reducing fibroblastic reactions. 
Table II

DISTRIBUTION OF PATIENTS ACCORDING TO THE PERIODS OF PREOPERATIVE PREPARATION FOR DERMAL PLASTY (IN DAYS).

\begin{tabular}{|c|c|c|c|c|c|c|c|c|}
\hline Earlycomplications & $\begin{array}{l}\text { First } \\
\text { group }(n=76)\end{array}$ & & $\begin{array}{l}\text { Second } \\
\text { group }(n=44)\end{array}$ & & $\begin{array}{l}\text { Comparison } \\
\text { group }(n=58)\end{array}$ & & $\operatorname{Total}(\mathrm{n}=178)$ & \\
\hline & Percentage & $\mathrm{R}$ & Percentage & $\mathrm{R}$ & Percentage & $\mathrm{R}$ & Percentage & $\mathrm{R}$ \\
\hline Edge necrosis of the flap & $7(9.2 \%)$ & 3 & $3(6.8 \%)$ & 3 & $8(13.8 \%)$ & 3 & $18(10.1 \%)$ & 3 \\
\hline Subflap seroma & $6(7.9 \%)$ & 4 & $4(9.1 \%)$ & 2 & $7(12.1 \%)$ & 4 & $17(9.6 \%)$ & 4 \\
\hline Postoperative suppuration of the wound & $14(18.4 \%)$ & 1 & $5(11.4 \%)$ & 1 & $15(25.9 \%)$ & 1 & $34(19.1 \%)$ & 1 \\
\hline Postoperative divergence of the edges of the wound & $8(10.5 \%)$ & 2 & $2(4.5 \%)$ & 4 & $9(15.5 \%)$ & 2 & $19(10.7 \%)$ & 2 \\
\hline Bleeding & $1(1.3 \%)$ & 5 & $1(2.3 \%)$ & 5 & $0(0 \%)$ & 5 & $2(1.1 \%)$ & 5 \\
\hline Total & $36(47.4 \%)$ & & $15(34.1 \%)$ & & $39(67.3 \%)$ & & $90(50.6 \%)$ & \\
\hline
\end{tabular}

\section{$K$. Timing of dermal plasty}

The main indicator of the effectiveness of preoperative preparation in our study is the timing of dermal plasty from the time of admission. The shortest periods of preoperative preparation were in the patients with pressure sores of the sacral area and amounted to $11.2 \pm 0.9$ days. The most time was spent for preparation of pressure sores of ischial tuberosity $-14.4 \pm 1.2$ days, which was associated with a complicated form of wounds, side pockets, osteomyelitis of the ischium bone. The use of NPWT in the second group reduced the preoperative period to $10.4 \pm 0.9$ days. The application of ozone therapy techniques allowed plastic surgery in the first group on $12.1 \pm 1.1$ days. The most prolonged was the preoperative period with the use of traditional methods $15.9 \pm 1.4$ days, and due to the preparation of pressure sores of the ischial tuberosity $-18.5 \pm 1.5$ days, with a significant difference $(\mathrm{p}<0.05)$.

Plastic closure of defects was carried out in all 178 injured: autodermoplasty with a split skin flap - $11(6.2 \%)$, plasty with local tissues $-46(25.9 \%)$, plasty with displaced flaps - 121 (67.9\%). The technique of a split dermatomic flap was used only in patients with the stage 3 pressure ulcers localized in the sacral area. Plastic surgery by local tissues was carried out in patients with deep pressure sores of small area, provided that there was sufficient mobility of the edges, most often in the area of ischial tuberosity, through extensive mobilization and use for the plasty of subcutaneous tissue and muscles. Plastic surgery with flaps was performed in 9 patients $(5.1 \%)$, V-Y plasty in 15 patients $(8.4 \%)$. Most often, plastic surgery was carried out using the displaced flaps by transposing the flap in 47 patients $(38.8 \%)$, rotating the flap in 26 patients $(21.5 \%)$ and using sliding plasty in 48 patients $(39.7 \%)$. Preference was given to full-layer skin-muscle flaps on the feeding stem $-88(72.7 \%)$.

\section{Postoperative complications}

Analysis of early postoperative complications on days 26 showed that they occurred in $90(50.6 \%)$ of the affected, almost in every second (Table 5). Most often, the early postoperative period was complicated by suppuration in $19.1 \%$, divergence of the edges of the wound in $10.7 \%$ and the edge necrosis of the flap in $10.1 \%$. In the second group, the smallest proportion of early complications was observed due to a decrease in the number of divergence of the wound edges to $4.5 \%$ and suppuration to $11.4 \%$. In cases of the edge necrosis of the flap, sutures were opened and repeated plasty was made with local tissues after complete demarcation and appearance of granulations.

During the long follow-up of the injured after the end of inpatient treatment, it was revealed that in the comparison group the different complications occurred in 19 (37.3\%) patients: fistula in the postoperative wound area $-11.8 \%$, relapse $-17.7 \%$, scar deformity $-7.8 \%$. Relapses of pressure sores appeared in 6 of 9 patients after autodermoplasty. A characteristic pattern of the appearance of fistulas in the area of ischial tuberosity was established in the patients who did not undergo a resection of the ischium because of mechanical loads on the operated site. The percentage of late complications was significantly less in the first group (13.6\%) and the lowest in the second group $(6.8 \%)$.

\section{CONCLUSION}

The use of the NPWT method in patients with pressure sores at the stage of preoperative preparation significantly increased the activity of local microcirculation, stimulated neoangiogenesis, promoted rapid bacterial decontamination and the appearance of morphological features typical for mature granulation tissue. This allowed for the plasty of defects to be carried out after $10.4 \pm 0.9$ days of admission. NPWT therapy had the lowest rate of early complications.

The determination of immunohistochemical markers CD34, CD68 reflects the degree of reparative processes in the wound. The increase in the density of CD34-expressed vessels and the quantitative index of CD68+ macrophages of $40.8 \pm 1.7$, with the minimal advantage of the functionally active macrophages are the objective criteria for the readiness of tissues to their plastic closure.

The method of selected pressure sore plasty consists in the use of displaced (local) musculocutaneous flaps with axial blood supply in combination with anatomical and functional resection of the underlying bones, which reduced the number of early postoperative complications in the first and second groups to $47.4 \%$ and $34.1 \%$, respectively, good and satisfactory results were achieved in 109 (90.8\%) of the patients.

\section{REFERENCES}


[1] L. Bublyk, G. Serbin, and Y. Titov, "Treatment and prophylaxis of bedsores in patients with traumatic disease of the spinal cord," Trauma, vol. 5, pp. 125-126, 2013.

[2] K. Oliinyk, "Problem of lack single algorithm prevention of bedsores according to the current requirements," Medsestrynstvo, vol. 4, pp. 21$22,2014$.

[3] A. Brusco, A. Khashchuk, O. Buryanov, and E. Shuvalov, "Morphological changes in the area of compressive trophic ulcers in patients with the consequences of complicated spinal injury," Ukrainian Morphological Almanac, vol. 2, pp. 12-16, 2012.

[4] D. Pasichniy, "Prognostic test for viability of the flap and preparation of tissues to italian plasty in defects of extremities," Clinical Surgery, vol. 4, pp. 49-52, 2014.

[5] T. Zvyagintseva and I. Khalin, "Metabolotropic therapy of chronic wounds," Apostrophe, pp. 18-29, 2011.

[6] I. Makagonov, A. Vergun, Y. Chulovskyj, S. Kit, and O. Vergun, "Authors views as to multimodality treatment of complicated bed-sores of the soft skin tissues from the standpoint of clinical strategies in the department of palliative care," Clinical and Experimental Pathology, vol. 15 , no. 3 , pp. $69-75,2016$

[7] O. Ponomarenko, "Differentiated approach to closing of wound surfaces trunk and extremities after its mechanical damage," Klinichna khirurhiia, no. 7, pp. 55-57, 2015.

[8] E. Fistal, "Treatment of wounds and their consequences: experience, results, prospects," Bulletin of Urgent and Rehabilitation Medicine, vol. 15, pp. 5-6, 2014.

[9] A. Kiyasov, S. Petrova, and N. Reichlin, "Methods of immunohistochemistry: Guidelines on immunohistochemical diagnosis of human tumors," pp. 15-38, 2000.

[10] J. C. Dumville, J. Webster, D. Evans, and L. Land, "Negative pressure wound therapy for treating pressure ulcers," The Cochrane Library, 2015.

[11] P. Zubarev and B. Rysman, "Ultrasonic cavitation and ozonization in the treatment of patients with purulent-necrotic complications of the diabetic foot syndrome," Vestnik of Surgery im. I. I. Grekova, vol. 170, no. 1, pp. 48-53, 2011.

[12] Y. Yarmolyuk, O. Buryanov, and N. Borzykh, "Modern technologies in staged surgical treatment of victims with gunshot fractures," Surgery of Ukraine, vol. 2, pp. 14-19, 2017.

[13] M. Kashtalyan, S. Tertishniy, and K. Masunov, "Use of vac-therapy in comprehensive treatment of gunshot wounds," Visnyk morsjkoji medycyny, vol. 2, pp. 187-192, 2016.

[14] R. Mikhayusov, "Experience of application of vacuum systems in the treatment of soft-tissue gunshot wounds," Clinical Surgery, vol. 6, pp. 61-64, 2016.

[15] R. Wang and Y. Feng, "Comparisons of negative pressure wound therapy and ultrasonic debridement for diabetic foot ulcers: a network meta-analysis," International journal of clinical and experimental medicine, vol. 8, p. 12548-12556, 2015. 